\title{
Efficiency of LH+EC Current Drive in Tokamaks Featuring an Internal Transport Barrier
}

\author{
P. R. da S. Rosa ${ }^{1}$, L. F. Ziebell ${ }^{2}$, C. I. de Oliveira $^{2}$, G. N. Jardim ${ }^{1}$, and A. K. Bonfim ${ }^{1}$ \\ ${ }^{1}$ Departamento de Física, UFMS, Caixa Postal 549, 79070-900, Campo Grande, MS, Brazil \\ 2 Instituto de Física, UFRGS, Caixa Postal 15051, 91501-970, Porto Alegre, RS, Brazil \\ Received on 27 May, 2005
}

\begin{abstract}
In the present paper we study the effects due to the occurrence of radial transport of particles in a tokamak on the efficiency of current drive due to combined action of lower hybrid waves and electron cyclotron waves, in the presence of an internal transport barrier. The results are obtained by numerical solution of the Fokker-Planck equation which rules the evolution of the electron distribution function. We assume that the radial transport of particles can be due to magnetic or to electrostatic fluctuations, and compare the two situations. In both cases the efficiency of current drive is shown to increase with the increase of the fluctuations which originate the transport. The current drive efficiency is shown to depend weakly on the radial position of the barrier, with a slightly more pronounced dependence in the case of magnetic fluctuations.
\end{abstract}

\section{INTRODUCTION}

It is nowadays well established that plasmas in tokamaks may feature regions of reduced transport known as transport barriers (TB). These TB may occur either near the plasma edge, when they are denoted as ETB, from edge transport barrier, or in more internal regions, when they are denoted as ITB, from internal transport barriers (ITB's). In both cases, the TB's are characterized by localized and significant reduction of the transport coefficients for particles, heat and momentum. Due to the presence of the barrier, accumulation of particles and energy is expected to occur in the region internal to the barrier, with the corresponding increase in confinement time. The detailed mechanisms leading to the formation of TB's, particularly to the formation of ITB's, are not completely understood, but for the purposes of the present paper it is sufficient to know that they appear in plasmas subject to a variety of heating schemes: ohmic, lower hybrid, ion cyclotron, electron cyclotron, and neutral beam injection [1-3].

In this paper we intend to discuss the effect of radial particle transport, in the presence of an ITB, on the efficiency of current drive generated by the combined effect of lower hybrid (LH) and electron cyclotron (EC) waves in tokamaks. The investigation utilizes quasilinear theory in order to follow the time evolution of the electron distribution function, using a slab geometry to describe the tokamak. Density and temperature modifications which may occur due to the radial transport are taken into account. Two different mechanisms causing radial transport will be considered, the electrostatic transport, which is expected to be more effective on low velocity particles, and the transport of magnetic origin, which is expected to be more active on high velocity particles. Although taking into account radial particle transport, we will be interested in the study of situations in which the loss of particles at the plasma edge can be considered negligible, motivated by the existence of experiments such those performed at the DIII-D tokamak [4], and such those with double transport barrier performed at the Alcator C-Mod tokamak [2]. Other current drive experiments featuring negligible loss of fast particles have also been realized in JT60U, under conditions very similar to those assumed in the present paper, with extrapo- lation to ITER conditions indicating even smaller fast particle losses [5]. In order to implement such conditions of good confinement, we assume Neumann boundary conditions in our numerical calculations, which guarantee zero particle flux at the edge. In experiments where such conditions of good confinement typical of $\mathrm{H}$-mode confinement are not satisfied, the loss of particles at the plasma edge may play significant and deleterious role on the current drive efficiency [6].

The present paper is related to two previous investigations which have been published as Ref. [7] and Ref. [8]. Ref. [7] had objectives similar to those of the present paper, both investigating the effect of radial transport and of the occurrence of ITB's on the combined current drive efficiency due to LH and EC waves. However, in Ref. [7] the propagation of LH waves was described by a cruder model, if compared with the model employed in the present investigation and also employed in Ref. [8], which considered the case of current generation by $\mathrm{LH}$ waves alone. According to the present more detailed model of $\mathrm{LH}$ propagation, the $\mathrm{LH}$ waves do not arrive to regions of the tokamak in which they could be present with the previous model. Significant differences occur in the profiles of LH power absorption and generated current, and therefore the effects of transport and of the presence of the ITB may be significantly modified, as demonstrated in the case of current drive with $\mathrm{LH}$ waves [8]. Another difference between the approach of Ref. [7] and that of the present paper is that in Ref. [7] the only kind of transport considered was of magnetic origin, while in the present paper we compare transport of magnetic and of electrostatic origin. The effects of transport, either magnetic or electrostatic, and of the ITB on the current drive by combined effects of LH and EC waves, under the conditions prescribed by an improved model describing LH waves, constitutes the main motivation for the present investigation.

The paper is organized as follows. Section II presents a very short account of the equations which are employed and the models which describe the tokamak and the wave-particle interaction. The numerical approach is described in Section III, which also shows the results found by numerical solution of the Fokker-Planck equation, as a function of several parameters. Section IV presents the conclusions and final remarks. 


\section{THE FOKKER-PLANCK EQUATION AND THE NUMERICAL SCHEME}

We start with a Maxwellian distribution function and solve the Fokker-Planck equation obtained from quasilinear theory. If we take into account $\mathrm{LH}$ and $\mathrm{EC}$ waves, collisions, and particle transport, the Fokker-Planck equation is written symbolically as follows

$$
\partial_{\tau} f=\left(\partial_{\tau} f\right)_{L H}+\left(\partial_{\tau} f\right)_{E C}+\left(\partial_{\tau} f\right)_{c o l}+\left(\partial_{\tau} f\right)_{t}
$$

where the subscripts in the right-hand side denote, from the left to the right, the effect of LH and EC waves, collisions and particle transport. $f=f(u, \mu, s, \tau)$ is the electron distribution function, $\tau$ is the time normalized to the collision time at the center of the slab, $u$ is the electron momentum normalized to the central thermal momentum at $\tau=0, \mu=\cos \theta$ is the cosine of the pitch angle $\theta$, and $s=x / a$ is the radial coordinate along the direction perpendicular to the ambient magnetic field $\mathbf{B}_{0}=$ $B_{0} \mathbf{e}_{z}$, normalized to the minor radius $a$.

The term related to LH waves may be given as follows,

$$
\left(\partial_{\tau} f\right)_{L H}=\partial_{u_{\|}}\left(D_{L H} \partial_{u_{\|}} f\right)
$$

where $D_{L H}$ is the diffusion coefficient in momentum space, and parallel means the direction parallel to the equilibrium magnetic field. The explicit expression for the coefficient $D_{L H}$ can be found in the literature and will not be repeated here, for the sake of economy of space [8-10]. The important point is that the LH diffusion coefficient depends on the quantity $S\left(s, \tau, N_{\|}\right)$, which represents the spectrum of the energy flux for LH waves. It is evaluated as follows. We assume

$$
S\left(s, \tau, N_{\|}\right)= \begin{cases}S_{0} & N_{1} \leq N_{\|} \leq N_{2} \\ 0 & N_{\|}<N_{1}, N_{\|}>N_{2},\end{cases}
$$

where $S_{0}$ is the intensity of LH waves at position $s$ at time $\tau$, and $N_{1}, N_{2}$ are the limits of the LH spectrum at each point in the slab. $N_{1}$ is the maximum between the lower caustic and the value of $N_{\|}$obtained from the accessibility condition defined by the coupling point between fast and slow modes. $N_{2}$ is the minimum between the upper caustic and $N_{\| L}$, which is a limitation due to Landau damping, approximately given by $n_{L} / \sqrt{T_{e}}$, where $n_{L} \simeq 6-7$. Due to the Landau resonance condition, there is a corresponding range of parallel velocities where the particles are in resonance with the waves. In order to avoid discontinuities which can be deleterious to the numerical solution, at the edges of the resonant region the diffusion coefficient connects to the adjacent non-resonant region in velocity space by means of Gaussian ramps whose half-width is the local thermal velocity [11].

These limits of the LH spectrum in $N_{\|}$-space are obtained from a well known propagation model which assumes several back and forth trips of the LH waves before complete absorption due to Landau damping $[8,11,12]$. The use of this propagation model is one of the main differences between the investigation developed in the present paper and the approach adopted in Ref. [7].

The quantity $S_{0}$ appearing in Eq. (3) can be obtained considering that, due to the fast movement of the electrons on the magnetic surface, the energy acquired by the electrons when passing through the region affected by the wave is rapidly spread to all the magnetic surface, and the average LH intensity at each magnetic surface can be given by $P_{L H}(s, \tau) /\left(4 \pi^{2}|s| a R\right)$, and where

$$
P_{L H}(s, \tau)=P_{L H}(a)\left(1-4 \pi^{2} R a^{2} \int_{1}^{s} d s^{\prime} s^{\prime} \rho_{L H}\left(s^{\prime}, \tau\right)\right),
$$

with

$$
\rho_{L H}(s, \tau)=1.5 \times 10^{-16} T_{e 0} \mathrm{v}_{e 0} \int d^{3} u \frac{u^{2}}{2}\left(\partial_{\tau} f\right)_{L H},
$$

being the density of absorbed LH power at each position in the slab, and where $v_{e 0}$ is the collision frequency at the plasma center. The numerical constants are such that the units of $\rho_{L H}$ are $\mathrm{W} / \mathrm{cm}^{3}$. A similar quantity, $\rho_{E C}(s, \tau)$, can be defined for the density of absorbed EC power at each position, depending on the term $\left(\partial_{\tau} f\right)_{E C}$.

The EC term, calculated for each slab position, can be written as

$$
\begin{aligned}
\left(\partial_{\tau} f\right)_{E C} & =\frac{1}{u_{\perp}}\left(y_{l} \partial_{u_{\perp}}+\frac{u_{\perp}}{\sqrt{\mu_{e 0}}} \partial_{u_{\|}} n_{\|}\right) \\
& \times\left[u_{\perp} D_{E C}\left(y_{l} \partial_{u_{\perp}}+n_{\|} \frac{u_{\perp}}{\sqrt{\mu_{e 0}}} \partial_{u_{\|}}\right) f\right] .
\end{aligned}
$$

In this equation $n_{\|}=n_{\|}(s)$ is the parallel wave number corrected for refraction effects along propagation,

$$
n_{\|}(x)=n_{0} \frac{R_{0}+a}{R_{0}+s a}
$$

where $n_{0}$ is the refraction index at the plasma edge, $R_{0}$ the major radius, $a$ the minor radius and $x$ the slab position. Here, perpendicular means the direction perpendicular to the ambient magnetic field. $y_{l}=l \omega_{c} / \omega_{E C}$, where $\omega_{c}$ is the local electron cyclotron angular frequency, $\omega_{E C}$ is the angular frequency of the EC waves and $l$ is the cyclotron harmonic, $\mu_{e 0}=m c^{2} / T_{e 0}$, with $T_{e 0}$ the central electron temperature at $\tau=0, m$ the electron mass and $c$ the speed of light. $D_{E C}$ is the diffusion coefficient in momentum space, which can be easily found in the literature [13-15]. The point to be emphasized is that the EC diffusion coefficient is averaged over the magnetic surfaces, assumed to be cylindrical, for simplicity. Although the ITB's are not typical of this kind of geometry, the magnetic surface only appears in the surface averaging of the EC diffusion coefficient, and for that purpose any surface shape with the same surface area would be equally effective, at least if the effect of trapped electrons is considered to be negligible, as in the present investigation, which deals with a large aspect ratio tokamak. Moreover, for the parameters to be used in the numerical investigation, the EC absorption occurs only in the low-field size, and therefore the actual shape of the whole surface area is not so significant for the evaluation of the EC absorption. The approximation of cylindrical magnetic surface therefore seems appropriated for the purposes of the present investigation. The EC diffusion coefficient depends on the spectrum of EC power on the magnetic surfaces. 
The spectrum is assumed to be Gaussian at the edge, and takes into account absorption self-consistently evaluated along the trajectory,

$$
\begin{aligned}
& P_{E C}\left(s, \tau, N_{\|}\right)=\frac{P_{E C}(a)}{\sqrt{\pi} \Delta n_{\|}} e^{-\frac{\left(n_{\|}-\bar{n}_{\|}\right)^{2}}{\left(\Delta n_{\|}\right)^{2}}} \\
& \times \exp \left(-\frac{2 \omega_{E C} a^{2}}{c} \int_{1}^{s} d s^{\prime} n_{\perp}^{\prime \prime}\left(s^{\prime}\right)\right),
\end{aligned}
$$

where $P_{E C}(a)$ is the EC power delivered by the antennas at the plasma edge, $\bar{n}_{\|}$is the parallel refraction index of the central ray of the wave packet, and $n_{\perp}^{\prime \prime}$ is the imaginary part of the perpendicular wave number, obtained from the dispersion relation for EC waves. Details upon the procedures for calculation of the components of the dielectric tensor and for the numerical solution of the dispersion relation can be found in the literature $[14,15]$.

The effect of collisions is introduced by the third term in the right-hand side of equation (1). We have used a linearized form which describes the interaction of fast electrons with body electrons and ions, for each slab position, which is also well-known and therefore will not be repeated here [7].

The last term in the right-hand side of equation (1) describes the transport of particles and has the following general form

$$
\left(\partial_{\tau} f\right)_{t}=\partial_{s}\left(D \partial_{s} f\right)
$$

$D$ being the particle diffusion coefficient, which can be given as follows [16-20],

$$
D=D_{m}+D_{e}=\frac{2 \pi q R_{0} c}{v_{e 0}}\left(\frac{\left|u_{\|}\right|}{\gamma \mu_{e 0}^{1 / 2}} \widetilde{b}^{2}+\frac{\gamma \mu_{e 0}^{1 / 2}}{\left|u_{\|}\right|} \widetilde{e}^{2}\right)
$$

where $D_{m}$ is the contribution to transport due to magnetic fluctuations, and $D_{e}$ is the contribution to transport when electrostatic fluctuations are present. For the numerical solution, we have assumed that the term due to electrostatic fluctuations goes smoothly to zero for $\left|u_{\|}\right|$well below the lower limit of the range of parallel velocities resonant with the LH and EC waves, therefore avoiding the divergence at $\left|u_{\|}\right|=0$ which appears in Eq. (8). The quantities $\widetilde{b} \equiv\left(B / B_{0}\right)$ and $\widetilde{e} \equiv\left(E / c B_{0}\right)$ give, respectively, the magnetic and electrostatic perturbation level of turbulence, normalized to the magnitude of the local ambient magnetic field, $B_{0}(s) . \gamma$ is the relativistic factor, and the quantity $q$ is the safety factor, assumed to be constant along the time evolution. This hypothesis is justified since the evolution occurs in the kinetic time scale, while experimental data show that the changes in the $q$ profile occurs in a longer time scale, of order of seconds $[1,21]$.

The safety factor $q$ is simulated in the present study by a parabolic profile which has its minimum value at position $s=0.5$, half-way between the center of the plasma and the plasma edge, as shown in Fig. 1b of Ref. [7]. It simulates the reversed $q$ profiles typically found in situations where there is ITB formation, since it has been found that these barriers form close to the position of the minimum of the $q$ profile $[1,22,23]$. In fact, recent studies indicate that the ITB usually occurs at the position of minimum $q$ or closer to the plasma center, but this is not an absolute restriction [3]. Therefore we consider in the numerical solution the canonical case in which the center of the ITB occurs at the position of the minimum $q$, but we also consider some cases where the barrier is displaced either toward the plasma center or toward the plasma edge, when investigating the effect of barrier position on the current drive efficiency.

The ITB is simulated by assuming that the level of magnetic (or electrostatic) turbulence is uniformly equal to $\widetilde{b}_{0}$ (or $\widetilde{e}_{0}$ ) everywhere except in the barrier region, where it is reduced down to a minimum value $\alpha \widetilde{b}_{0}$ (or $\alpha \widetilde{e}_{0}$ ). The barrier is centered at $s=s_{b}$, with nondimensional half-width $\beta=0.15$. Specifically, we assume a parabolic reduction of the magnetic turbulence level at the barrier position (for $\left.s_{b}-\beta<s<s_{b}+\beta\right)$, such that $\widetilde{b}=\widetilde{b}_{0}\left(a_{1}+a_{2} s+a_{3} s^{2}\right)$ and $\widetilde{e}=\widetilde{e}_{0}\left(a_{1}+a_{2} s+a_{3} s^{2}\right)$, where the $a_{i}$ are constants [7]. As boundary conditions of the barrier region in the case of magnetic fluctuations we assume $\widetilde{b}=\widetilde{b}_{0}$ at $s=s_{b} \pm \beta$ and $\widetilde{b}=\alpha \widetilde{b}_{0}$ at $s=s_{b}$. In the case of electrostatic fluctuations, similarly, we assume $\widetilde{e}=\widetilde{e}_{0}$ at $s=s_{b} \pm \beta$ and $\widetilde{e}=\alpha \widetilde{e}_{0}$ at $s=s_{b}$. The resulting profile of magnetic and electrostatic turbulence is the same as the profile of magnetic turbulence which can be seen in Figure 1a of Ref. [7], for several values of $\alpha$ and $\beta=0.15$. The model describes a situation where an ITB is already formed when RF waves are injected in the plasma in order to generate current. Modifications on the barrier profile due to RF waves are not taken into account in this scenario.

For the magnetic field, initial density and temperature profiles we make use of the following expressions

$$
\begin{aligned}
& B_{0}(s)=B_{0}(0)\left(1+s \frac{a}{R}\right)^{-1} \\
& n_{e}(s, \tau=0)=\left(n_{e 0}-n_{e a}\right)\left(1-s^{2}\right)+n_{e a} \\
& T_{e}(s, \tau=0)=\left(T_{e 0}-T_{e a}\right)\left(1-s^{2}\right)^{2}+T_{e a}
\end{aligned}
$$

where the indexes 0 and $a$ indicate, respectively, values taken at the plasma center and plasma edge. Along the time evolution the density and the temperature are updated at each collision time, to take into account modifications in the profiles caused by radial transport.

The quasilinear equation, Eq. (1), is solved using the ADI method (implicit in alternate directions) in order to obtain the time evolution of the electron distribution function. We assume a grid with $151 \times 31 \times 81$ points in the $u \times \mu \times s$ space, with $0 \leq u \leq 12,-1 \leq \mu \leq 1$ and $-1 \leq s \leq 1$. The time variable is discretized assuming $\Delta \tau=0.01$. Neumann boundary conditions are assumed at plasma edge, which imply that the total number of particles initially present, $N_{0}$, is kept constant along the calculations. This condition is imposed as a normalization condition for the density profile: $\int d^{3} s n_{e}(s, \tau)=N_{0}$. 


\section{NUMERICAL RESULTS}

For the numerical analysis, we assume the following parameters, typical of medium-size tokamaks, chosen in order to guarantee total $\mathrm{LH}$ power absorption, for the assumed profiles: $n_{e 0}=6.0 \times 10^{19} \mathrm{~m}^{-3}, T_{e 0}=3.0 \mathrm{keV}, B_{0}(0)=2.65 \mathrm{~T}, a=0.2$ $\mathrm{m}, R=1.0 \mathrm{~m}$. For the LH waves, we consider $P_{L H}(a, t)=0.5$ MW, and choose $N_{\| 0}=2.3$ and $f_{L H}=3.37 \mathrm{GHz}$. For most of the applications, unless explicitly stated, we use $s_{b}=0.5$, $\alpha=0.125$ and $\beta=0.15$.

As we have already discussed, at each point in the slab there is a range of values of the parallel refraction index which is available to the LH waves, limited by $N_{1}$ and $N_{2}$. There is a corresponding range of resonant velocities, which can be seen, for instance, in Fig. 1 of Ref. [8], for the parameters listed in the previous paragraph, as a function of position inside the slab. The range of resonant velocities is position-dependent, although nearly uniform in a significant part of the slab. For instance, for the parameters listed in the previous paragraph, the resonant velocities nearly span the range between $u_{\|} \simeq 3.3$ and $u_{\|} \simeq 6.0$, in the region of the slab where most of the LH wave power is deposed, around $s \simeq 0.5$ [8].

For the EC waves, we consider the extraordinary mode, with frequency $f_{E C}=140 \mathrm{GHz}$, injected at an angle $\psi=20^{\circ}$ relative to the perpendicular to the ambient magnetic field, with $\Delta \psi=3^{\circ}$ as the half-width of the spectrum. As a consequence, $\bar{n}_{\|}=\sin \psi \simeq 0.342$, and $\Delta n_{\|} \simeq 0.052$. For most of the applications, unless explicitly stated, we use $P_{E C}=0.5$ MW.

Fig. 1(a) shows the density of $L H$ and $E C$ power absorbed as a function of position in the plasma slab, at $\tau=80$, for the case of magnetic transport with $\widetilde{b}_{0}=3.0 \times 10^{-5}$ and $\widetilde{e}_{0}=0.0$, and barrier depth given by $\alpha=0.125$. It is also shown the density of LH power at $\tau=50$ and at $\tau=0$, for the same case. The curve for $\tau=50$ shows that at the end of the LH phase the LH power is mainly deposed near the position $s=0.4$, slightly displaced toward the plasma edge as compared to the initial profile of deposition. At $\tau=80$, the deposition of $\mathrm{LH}$ waves has moved further outward, toward the position $s=0.5$, with the profile slightly broadened when compared to the situation at $\tau=50$. The EC absorption occurs in a region narrower than the LH absorption, around position $s \simeq 0.45$.

Fig. 1(b) shows curves corresponding to those of Fig. 1(a), but obtained considering the absence of the ITB. The results displayed in panel (b) of the figure are very similar to those of panel (a), with the only difference that the profiles of LH absorption at $\tau=50$ and at $\tau=80$ are very slightly more broadened in the case of absence of barrier than in the case with the barrier.

Fig. 1(c) shows curves corresponding to those of Figs. 1(a) and $1(\mathrm{~b})$, but obtained ignoring the effect of transport. We observe that the peak of LH absorption is moved outward between $\tau=0$ and $\tau=50$, even in the absence of transport. This occurs due to the formation of the electron tail in the electron distribution and the consequent modification of the absorption of the wave. This process has already attained steady state at $\tau=50$, and is not affected by EC waves, since the curves for LH absorption at $\tau=50$ and $\tau=80$ are nearly the same.
However, when comparing the case without transport shown in Fig. 1(c) with the cases with transport shown in Figs. 1(a) and 1(b), we observe that the effect of transport is revealed in the broadening of the LH profile. The presence of transport also allows for synergy effects between LH and EC waves, revealed by the slight modification in the LH absorption profile which occurs between $\tau=50$ and $\tau=80$, appearing in Figs. 1 (a) and 1(b).
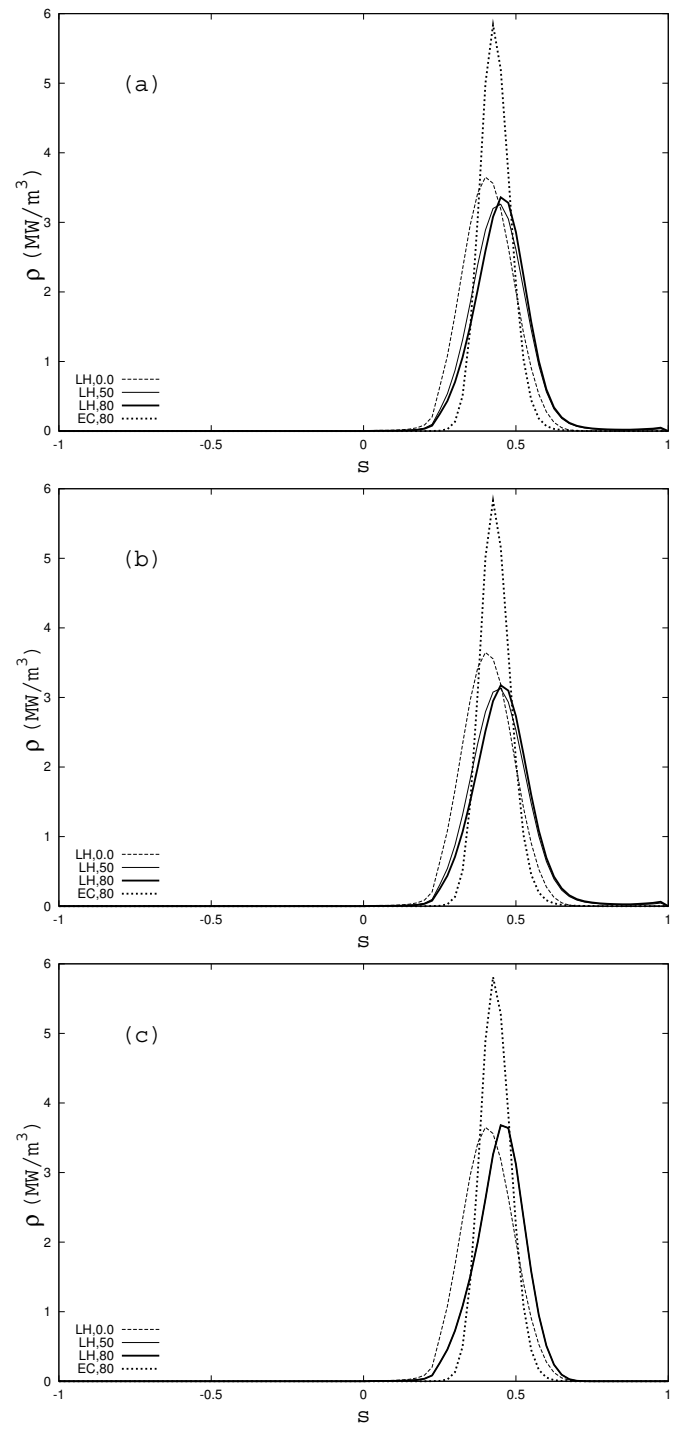

FIG. 1: $\rho_{L H}$ and $\rho_{E C}$ vs $s$, for $P_{L H}(a, t)=0.5 \mathrm{MW}$ and $P_{E C}(a, t)=$ 0.5 MW. (a) The case of magnetic transport, $\widetilde{b}_{0}=3.0 \times 10^{-5}$ and $\widetilde{e}_{0}=0.0$. The ITB is located at $s_{b}=0.5$, with $\alpha=0.125$, and $\beta=$ 0.15 . (b) The case of magnetic transport, $\widetilde{b}_{0}=3.0 \times 10^{-5}$ and $\widetilde{e}_{0}=$ 0.0 , without the presence of the ITB. (c) The case without effects of transport. The curves shown in each panel are: $\rho_{L H}$ at $\tau=0.0$ (thin dashed line); $\rho_{L H}$ at $\tau=50.0$ (thin line); $\rho_{L H}$ at $\tau=80.0$ (thick line); $\rho_{E C}$ at $\tau=80.0$ (thick dotted line). The parameters are characteristic of a medium size tokamak. $n_{e 0}=6.0 \times 10^{19} \mathrm{~m}^{-3}, T_{e 0}=3.0 \mathrm{keV}$, $B_{0}(0)=2.65 \mathrm{~T}, a=0.2 \mathrm{~m}, R=1.0 \mathrm{~m}$. For the LH waves, $N_{\| 0}=2.3$, $f_{L H}=3.37 \times 10^{9} \mathrm{~Hz}$, and $n_{L}=6.5$. 
Fig. 2 shows the current density $J$ vs position in the plasma slab $s$, for several values of $\tau$. Panel (a) shows the case of magnetic transport with $\widetilde{b}_{0}=3.0 \times 10^{-5}$ and $\widetilde{e}_{0}=0.0$, panel (b) shows the case of electrostatic transport with $\widetilde{b}_{0}=0.0$ and $\widetilde{e}_{0}=0.5 \times 10^{-5}$, and panel (c) the case without transport. Other parameters are the same as in Fig. 1. It is noticeable the fast increase of current density after the onset of EC waves, which occurs at $\tau=50$. It is also noticeable in panels (a) and (b) the spread of the profile of current density due to the radial transport, when compared with the case without transport in panel (c).

The spread is slightly more pronounced in the case of magnetic transport, panel (a), than in the case of electrostatic transport, panel (b). In both cases, magnetic transport and electrostatic transport, the position of the peak of current density is not noticeable moved by the introduction of EC waves, and is not moved by the effect of transport. A rough quantitative analysis can be as follows. In the case without transport, depicted in panel (c), the peak of current density at $\tau=80$ is nearly $6.1 / 3.7 \simeq 1.65$, or $65 \%$ higher than the peak at the end of the LH only phase, at $\tau=50$. Taking into account the effect of transport of magnetic origin, as in panel (a), significant spread occurs, but the ratio between the peaks of current density at $\tau=80$ and at $\tau=50$ remain nearly the same, given by $4.5 / 2.8 \simeq 1.61$. In the case of electrostatic transport, shown in Fig. 2(b), the peak of current density at $\tau=80$ is nearly $4.9 / 3.1 \simeq 1.58$, or $58 \%$ higher than the peak at the end of the LH only phase, at $\tau=50$. The peaks are higher than the corresponding peaks in panel (a), obtained in the case of magnetic transport, and one notices less spread toward the plasma edge. It appears that the electrostatic transport at this turbulence level is less efficient in spreading the profile of LH current density than the magnetic transport. The reason is that the magnetic transport is more effective for high-energy particles than the electrostatic transport, according to the model utilized. The magnitudes of the electrostatic and magnetic fluctuation levels assumed for Figs. 2(a) and 2(b) are within the range of experimental values [20, 24, 25], and have already been utilized in a previous analysis on transport effects on $\mathrm{LH}$ current generation [8].

Fig. 3(a) shows the current generated as a function of time, displaying the case without transport and the cases of magnetic transport, with $\widetilde{b}_{0}=3.0 \times 10^{-5}$ and $\widetilde{e}_{0}=0.0$, and electrostatic transport, with $\widetilde{b}_{0}=0.0$ and $\widetilde{e}_{0}=0.5 \times 10^{-5}$. It is seen that the presence of transport contributes to the increase of the generated current. It is also seen that the effect of electrostatic transport on the generated current is remarkably similar to the effect of magnetic transport. In Fig. 3(a) and in many of the following figures, the case of magnetic transport is indicated by MG, and the case of electrostatic transport is indicated by ES.

The efficiency of current drive may be measured by the nondimensional parameter

$$
\eta=33 \frac{|I| R n_{\text {ave }}}{\left(P_{L H}+P_{E C}\right) T_{\text {ave }}},
$$

where $n_{\text {ave }}$ is the electron density expressed in units of $10^{20}$ $\mathrm{m}^{-3}$, averaged along the radial coordinate, and $T_{a v e}$ is the
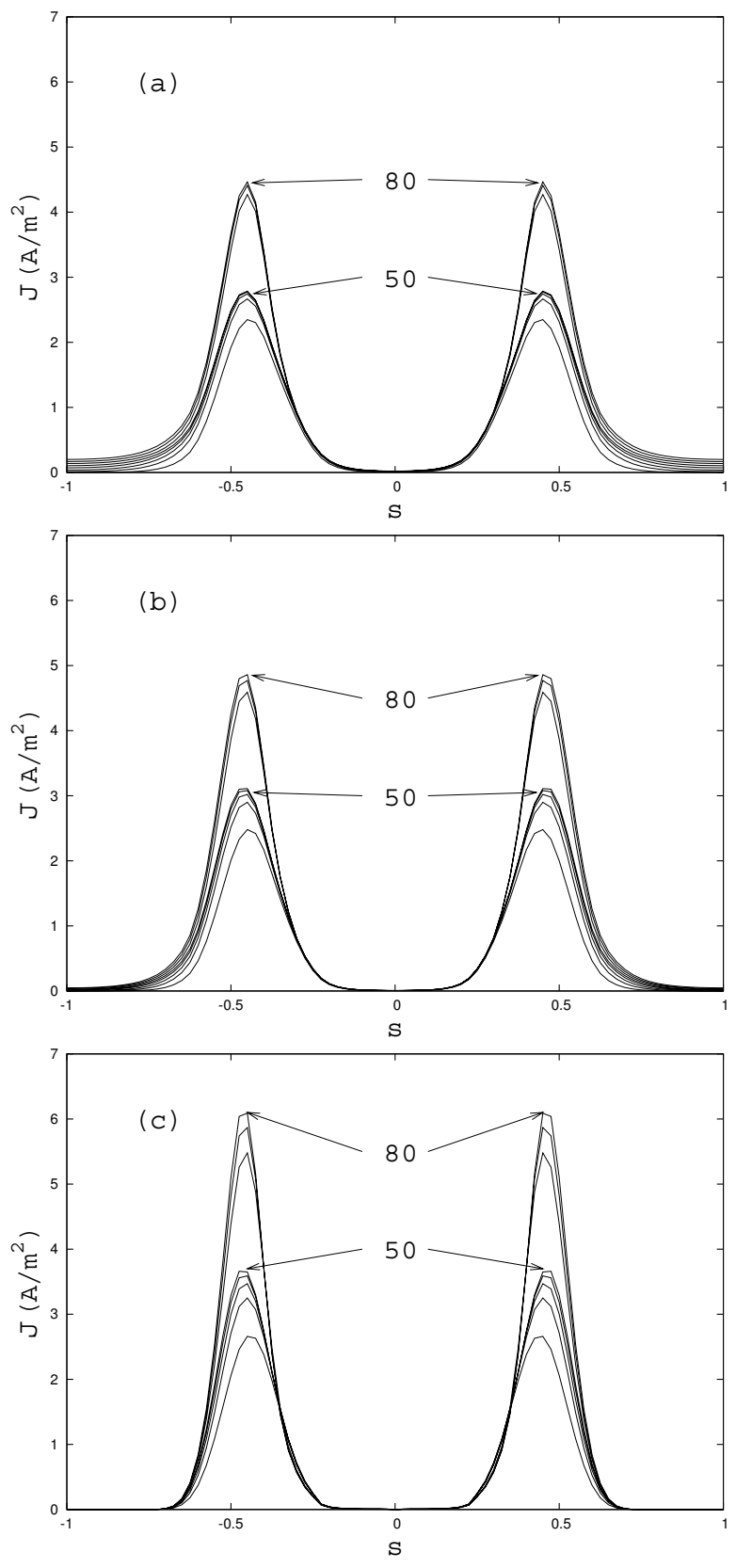

FIG. 2: $J$ vs $s$ for several values of $\tau$. The lines show the values of $J$ at $\tau=10,20,30,40,50,60,70$, and 80 . (a) The case of magnetic transport with $\widetilde{b}_{0}=3.0 \times 10^{-5}$; (b) The case of electrostatic transport with $\widetilde{e}_{0}=0.5 \times 10^{-5}$; (c) the case without transport. Other parameters are the same as in Fig. 1.

electron temperature expressed in $\mathrm{keV}$, also averaged along the radial coordinate [26].

Fig. 3(b) shows the current drive efficiency as a function of time, displaying the case without transport and the cases of magnetic transport, with $\widetilde{b}_{0}=3.0 \times 10^{-5}$ and $\widetilde{e}_{0}=0.0$, and electrostatic transport, with $\widetilde{b}_{0}=0.0$ and $\widetilde{e}_{0}=0.5 \times 10^{-5}$. It is seen that the presence of transport contributes to increase the efficiency of current drive, when compared to the case with- 

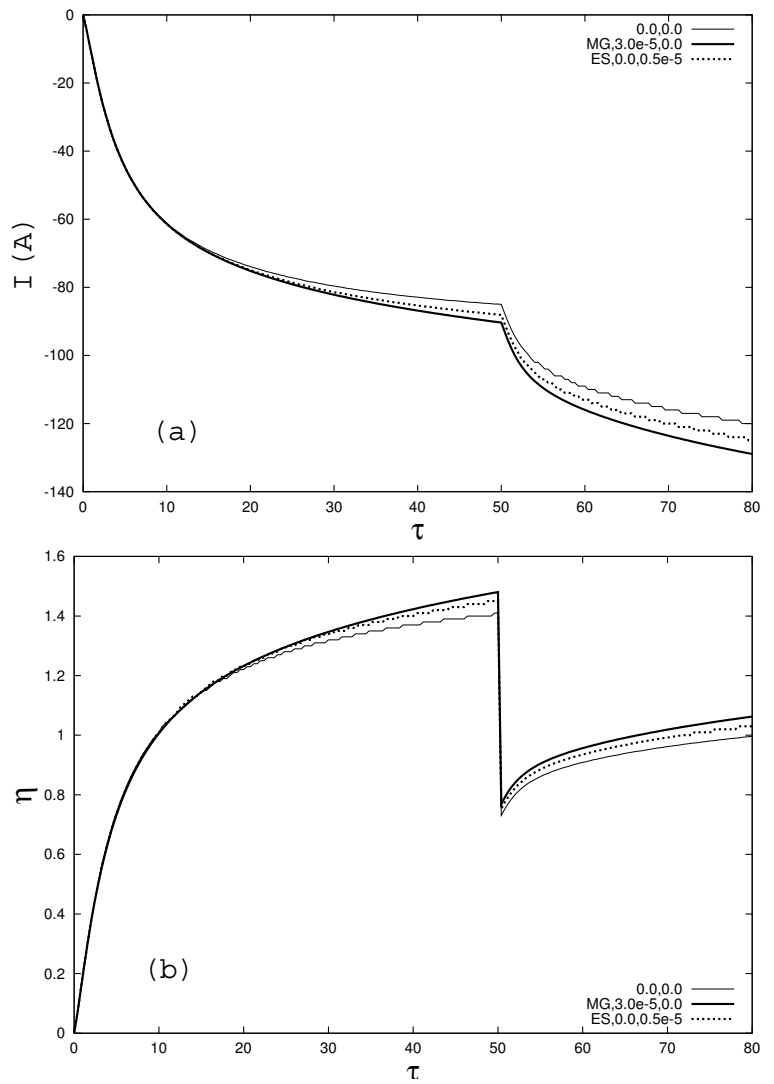

FIG. 3: (a) Current $I$ vs. normalized time $\tau$. (b) Current drive efficiency $\eta$ vs. normalized time $\tau$. The curves shown in each panel are: the case without transport (thin line); magnetic transport, with $\widetilde{b}_{0}=3.0 \times 10^{-5}$ and $\widetilde{e}_{0}=0.0$ (thick line); electrostatic transport, with $\widetilde{b}_{0}=0.0$ and $\widetilde{e}_{0}=0.5 \times 10^{-5}$ (thick dotted line). Other parameters are the same as in Fig. 1.

out transport. It is also seen that the effect of electrostatic transport on the efficiency of current drive is remarkably similar to the effect of magnetic transport. Although the current generated immediately increases after the start of EC waves, the overall efficiency decreases with the introduction of EC waves, comparing with the efficiency of the LH waves alone. After the sudden decrease which occurs at the onset of EC waves, the efficiency slowly increases again, but for the parameters utilized do not attain the same level attained with the LH waves alone.

Fig. 4(a) shows the current drive efficiency $\eta$ vs magnetic fluctuation level $\widetilde{b}_{0}$ ranging from $\widetilde{b}_{0}=0.0$ up to $\widetilde{b}_{0}=$ $5.0 \times 10^{-5}$, for $\widetilde{e}_{0}=0.0$, at $\tau=50$ and at $\tau=80$. Fig. $4(\mathrm{~b})$ shows the current drive efficiency $\eta$ vs electrostatic fluctuation level $\widetilde{e}_{0}$ ranging from $\widetilde{e}_{0}=0.0$ up to $\widetilde{e}_{0}=1.0 \times 10^{-5}$, for $\widetilde{b}_{0}=0.0$, at $\tau=50$ and at $\tau=80$. Other parameters are the same as in Fig. 1. Figs. 4(a) and (b) show that the electrostatic turbulence with $\widetilde{e}_{0}=1.0 \times 10^{-5}$ produces similar effect on the current drive efficiency as the magnetic turbulence with $\widetilde{b}_{0}=5.0 \times 10^{-5}$. Both in the cases of electrostatic and mag-
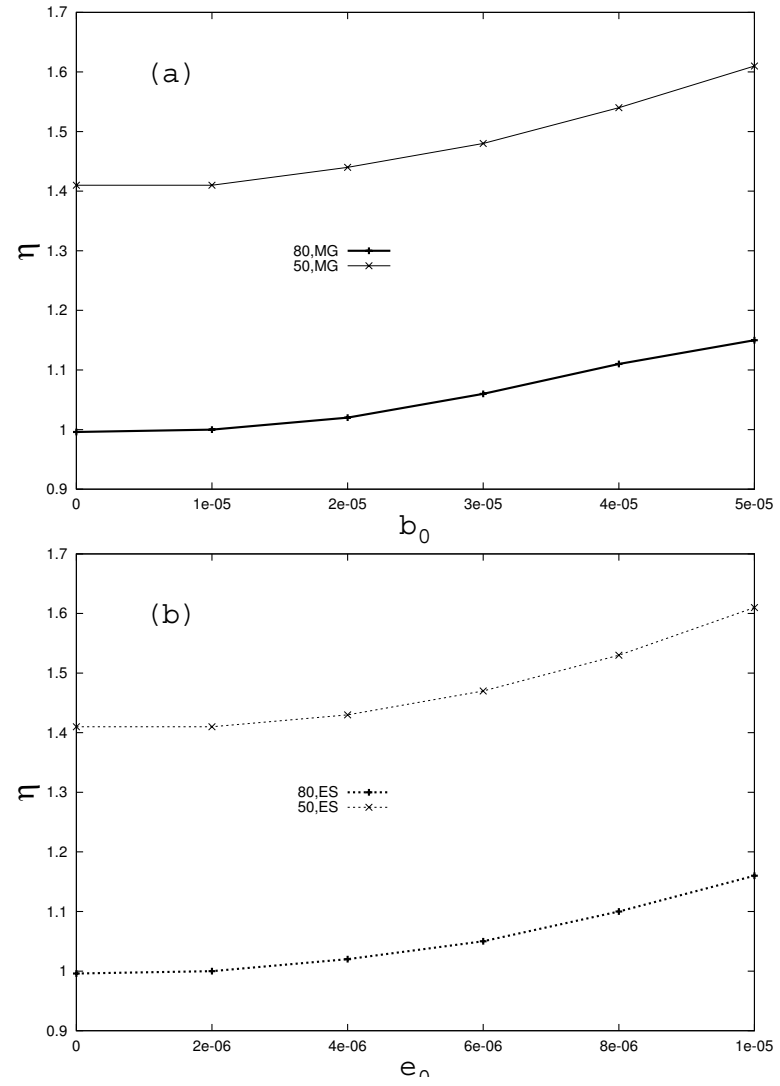

FIG. 4: (a) Current drive efficiency $\eta$ vs magnetic fluctuation level $\widetilde{b}_{0}$, for $\widetilde{e}_{0}=0.0$, at $\tau=50$ (thin line) and at $\tau=80$ (thick line). (b) Current drive efficiency $\eta$ vs electrostatic fluctuation level $\widetilde{e}_{0}$, for $\widetilde{b}_{0}=0.0$, at $\tau=50$ (thin dotted line) and at $\tau=80$ (thick dotted line). Other parameters are the same as in Fig. 1.

netic transport, the efficiency at $\tau=80$ is nearly $70 \%$ of the efficiency at the end of the LH phase, at $\tau=50$. It is also seen that the dependence of the current drive efficiency on the turbulence level, in the range considered, is nearly the same in the cases of magnetic and electrostatic transport.

The increase of the current drive efficiency with the increase of the turbulence level may be explained by the following mechanism. Fast particles are radially moved towards less dense regions of the tokamak by effect of the transport. In these regions the slow-down time due to collisions is larger, therefore the level of generated current is increased.

In Ref. [8] we have verified the consistency of this explanation by considering a situation with different plasma densities, keeping the same profiles and all other parameters. The argument can be illustrated with the results shown in Fig. 7 of Ref. [8], which shows that for increasing electron density the current drive efficiency is reduced. It also shows that the positive contribution of the transport to the current drive efficiency is more significant for smaller electron densities. Both findings are in accordance with the explanation given in the previous paragraph.

The effect of the position of the center of the ITB can be 


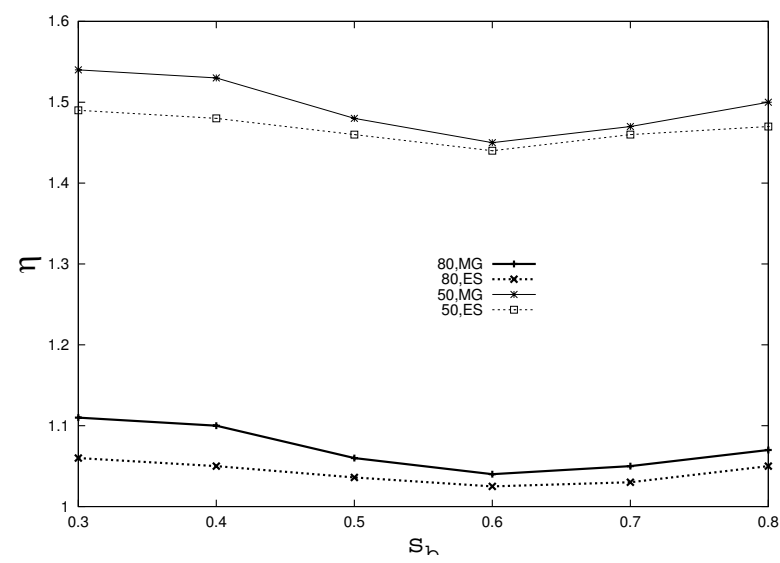

FIG. 5: Current drive efficiency $\eta$ vs position of the transport barrier, at $\tau=50$ and $\tau=80$; the two upper curves are for $\widetilde{b}_{0}=3.0 \times 10^{-5}$ and $\widetilde{e}_{0}=0.0$ (thin line) and for $\widetilde{b}=0.0$ and $\widetilde{e}=0.5 \times 10^{-5}$ (thin dotted line), respectively, at $\tau=50$. The lower curves are for $\widetilde{b}_{0}=$ $3.0 \times 10^{-5}$ and $\widetilde{e}_{0}=0.0$ (thick line) and for $\widetilde{b}=0.0$ and $\widetilde{e}=0.5 \times$ $10^{-5}$ (thick dotted line), respectively, at $\tau=80$. Other parameters are the same as in Fig. 1.

seen in Fig. 5, which shows the current drive efficiency $\eta$ vs position of the ITB, at $\tau=50$ and $\tau=80$. The two upper curves are for $\widetilde{b}_{0}=3.0 \times 10^{-5}$ and $\widetilde{e}_{0}=0.0$ and for $\widetilde{b}=0.0$ and $\widetilde{e}=0.5 \times 10^{-5}$, respectively, at $\tau=50$. The lower curves are for the same parameters, at $\tau=80$. Other parameters are the same as in Fig. 1. It is seen that, both at the end of the LH phase and at the end of the phase with combined effect of LH and EC waves, the efficiency is a minimum for $s_{b}=0.6$, increasing when the barrier is closer to the center of the slab and when the barrier is closer to the plasma edge. The explanation can be as follows. As seen in Fig. 2, the LH waves generate current around $s_{b}=0.5$. The radial diffusion toward the plasma edge tends to produce increase of the current. If the ITB is localized at $s_{b} \leq 0.5$, it does not hinder very much the diffusion toward the edge, and the current indeed increases. If the ITB is localized near $s_{b}=0.6$, it stands in the way of the radial diffusion between the region of energy deposition of LH waves and more external regions, and consequently the efficiency of current generation is decreased. However, if the ITB is closer to the edge, its effect on the particle diffusion starts becoming less important, since significant diffusion already occurred up to the position of the barrier. The efficiency of current generation may therefore increase by effect of the diffusion of particles from $s \simeq 0.5$ toward the position of the barrier. Fig. 5 indicates that this effect of the position of the barrier is more pronounced in the case of magnetic fluctuations than in the case of electrostatic fluctuation, both at $\tau=50$ and at $\tau=80$. Regarding the effect of the position of the barrier, the combined action of $\mathrm{LH}+\mathrm{EC}$ waves does not change appreciably the behavior observed with LH waves alone [8]

The effect of the barrier depth is investigated in Fig. 6, which shows the current drive efficiency $\eta$ vs $\alpha$, parameter related to the depth of the ITB, at $\tau=50$ and $\tau=80(\alpha=1$

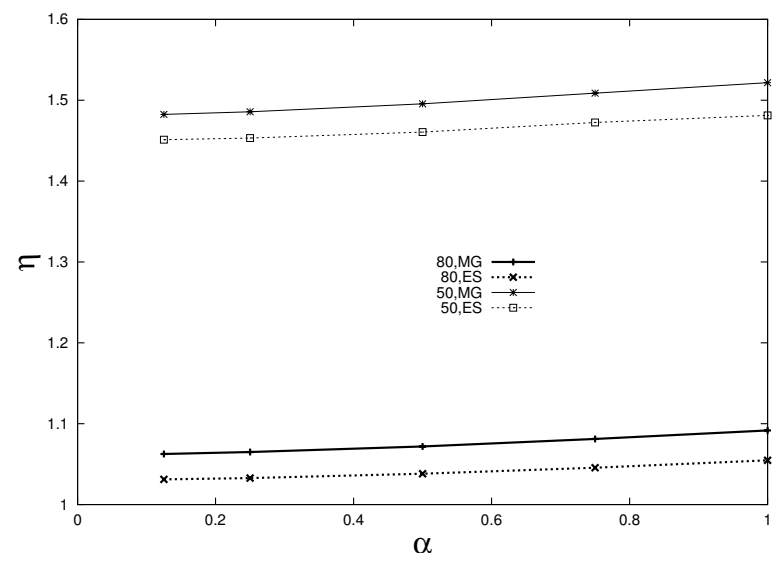

FIG. 6: Current drive efficiency $\eta$ vs depth of the transport barrier, at $\tau=50$ and $\tau=80$ (the barrier depth decreases toward the right); the two upper curves are for $\widetilde{b}_{0}=3.0 \times 10^{-5}$ and $\widetilde{e}_{0}=0.0$ (thin line) and for $\widetilde{b}=0.0$ and $\widetilde{e}=0.5 \times 10^{-5}$ (thin dotted line), respectively, at $\tau=50$. The two lower curves are for $\widetilde{b}_{0}=3.0 \times 10^{-5}$ and $\widetilde{e}_{0}=0.0$ (thick line) and for $\widetilde{b}=0.0$ and $\widetilde{e}=0.5 \times 10^{-5}$ (thick dotted line), respectively, at $\tau=80$. Other parameters are the same as in Fig. 1 .

represents the case without barrier). The two upper curves are for $\widetilde{b}_{0}=3.0 \times 10^{-5}$ and $\widetilde{e}_{0}=0.0$ and for $\widetilde{b}=0.0$ and $\widetilde{e}=$ $0.5 \times 10^{-5}$, respectively, at $\tau=50$. The lower curves are for the same parameters, at $\tau=80$. Other parameters are the same as in Fig. 1. It is seen that the current drive efficiency is almost independent of the barrier depth, featuring a very slight increase when the barrier depth is reduced until vanishing.

Fig. 7 shows the current drive efficiency $\eta$ vs EC power in MW, at $\tau=50$ and $\tau=80$. The lower curves are for $\widetilde{b}_{0}=3.0 \times 10^{-5}$ and $\widetilde{e}_{0}=0$, and for $\widetilde{e}_{0}=0.50 \times 10^{-5}$ and $\widetilde{b}_{0}=0$, at $\tau=80$. The curve in the upper part of the plot shows the value of $\eta$ at $\tau=50$, before the onset of EC waves. Other parameters are the same as in Fig. 1. It is seen that the efficiency of the current drive at $\tau=80$ decreases with the increase of the EC power, with the same rate of decrease in the cases of magnetic and electrostatic transport. The time evolution of the efficiency, which leads to the outcome appearing in Fig. 7, appear in Fig. 8, for $\tau$ ranging between 0 and 80, for several values of the EC power.

Although the efficiency of the current drive decreases with the increase of the EC power, the combined efficiency of LH and EC power is larger than the summation of the separated efficiencies due to LH and EC waves, as shown in Fig. 9, which displays the relative efficiency,

$$
\eta_{\text {relat }}=\frac{\eta_{L H+E C}}{\eta_{L H}+\eta_{E C}} \simeq \frac{I_{L H+E C}}{I_{L H}+I_{E C}},
$$

for the case of $P_{L H}=0.5 \mathrm{MW}$ and $P_{E C}=0.25 \mathrm{MW}, 0.50 \mathrm{MW}$, $0.75 \mathrm{MW}$, and $1.00 \mathrm{MW}$. It is seen that immediately after the onset of EC waves there is a reduction of the efficiency, but after a few collision times the combined efficiency becomes larger than the separated efficiencies, indicating a synergistic effect $[11,27-29]$. At $\tau=80$, Fig. 9 shows that in the case of 


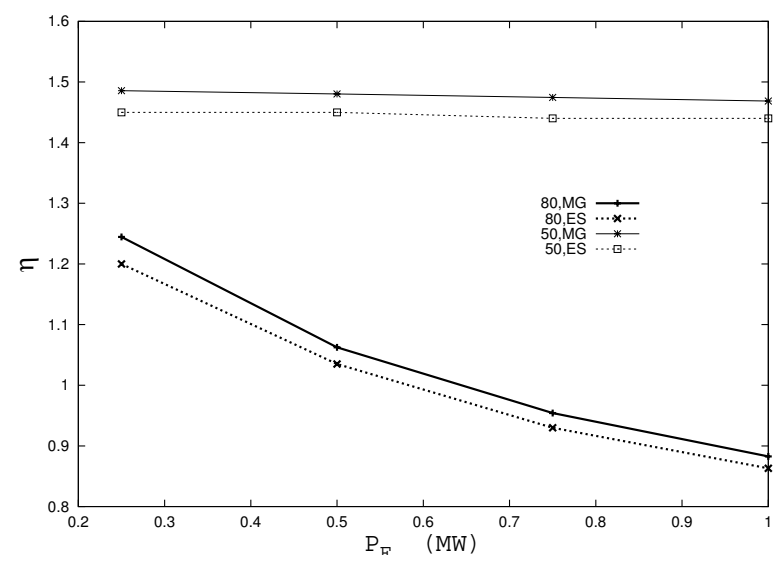

FIG. 7: Current drive efficiency $\eta$ vs EC power in MW, at $\tau=50$ and $\tau=80$; the two upper curves are for $\widetilde{b}_{0}=3.0 \times 10^{-5}$ and $\widetilde{e}_{0}=0.0$ (thin line) and for $\widetilde{b}=0.0$ and $\widetilde{e}=0.5 \times 10^{-5}$ (thin dotted line), respectively, at $\tau=50$. The two lower curves are for $\widetilde{b}_{0}=3.0 \times 10^{-5}$ and $\widetilde{e}_{0}=0.0$ (thick line) and for $\widetilde{b}=0.0$ and $\widetilde{e}=0.5 \times 10^{-5}$ (thick dotted line), respectively, at $\tau=80$. Other parameters are the same as in Fig. 1.

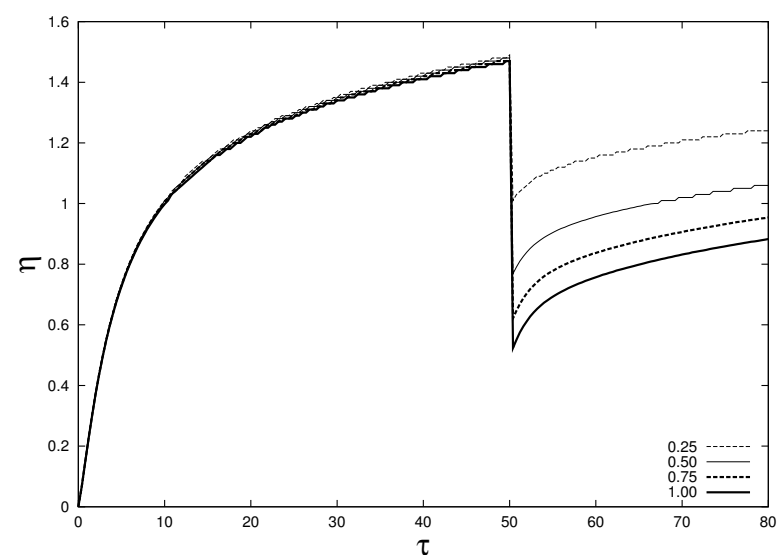

FIG. 8: Current drive efficiency $\eta$ vs $\tau$, for several values of EC power, in the case of magnetic transport, with $\widetilde{b}_{0}=3.0 \times 10^{-5}$ and $\widetilde{e}_{0}=0 . P_{E C}=0.25 \mathrm{MW}$ (thin dashed line), $0.50 \mathrm{MW}$ (thin line), 0.75 MW (thick dashed line), and 1.00 MW (thick line). Other parameters are the same as in Fig. 1.

greater EC power considered, which is twice the LH power, the combined efficiency is tending to an asymptotic increase of approximately $12 \%$, similar to the increase obtained in the case of $P_{E C}=2 P_{L H}$, in Ref. [7]. Although in Ref. [7] the model utilized for the propagation of LH waves was different from the model utilized here, in both case some spatial superposition between the absorption profiles of LH and EC waves was obtained. The magnitude of the synergistic effect obtained is relatively small. The reason is the following. Although the spatial regions of EC and LH absorption are nearly coincident, the superposition of effects in momentum space is not very large, for the parameters utilized. At the position of

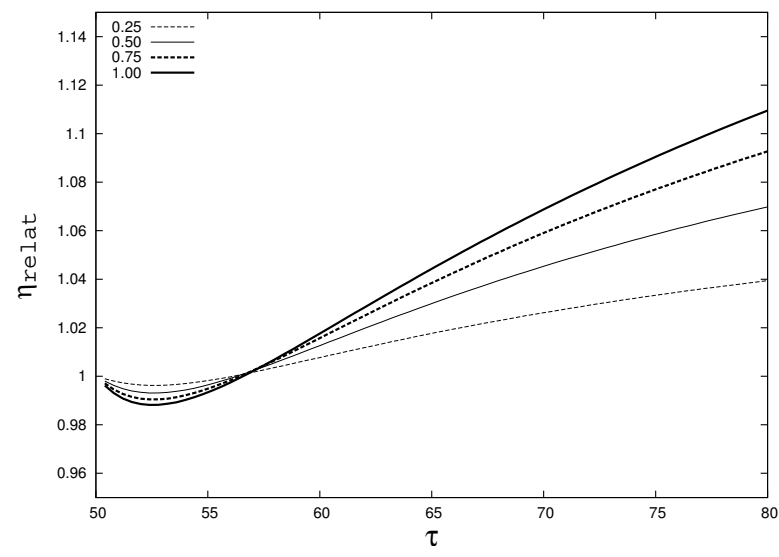

FIG. 9: Time evolution of the relative efficiency of current generation, $\eta_{\text {relat }}$, in the case of magnetic transport, with $\widetilde{b}_{0}=3.0 \times 10^{-5}$ and $\widetilde{e}_{0}=0$. The curves are obtained with $P_{L H}=0.50 \mathrm{MW}$, and $P_{E C}=$ $0.25 \mathrm{MW}$ (thin dashed line), $0.50 \mathrm{MW}$ (thin line), $0.75 \mathrm{MW}$ (thick dashed line), and 1.00 MW (thick line). Other parameters are the same as in Fig. 1.

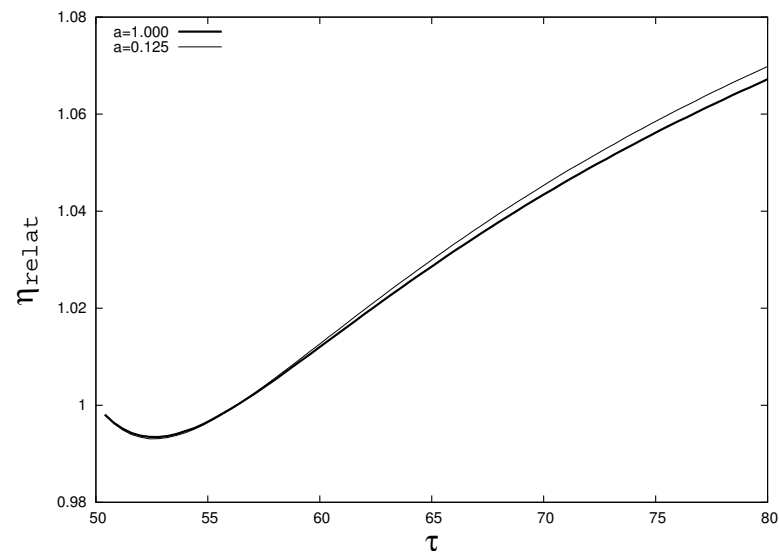

FIG. 10: Time evolution of the relative efficiency of current generation, $\eta_{\text {relat }}$, in the case of magnetic transport, with $\widetilde{b}_{0}=3.0 \times 10^{-5}$ and $\widetilde{e}_{0}=0$. The curves are obtained with $P_{L H}=0.50 \mathrm{MW}$, and show the cases $\alpha=0.125$ (thin line) and $\alpha=1.0$ (thick line). Other parameters are the same as in Fig. 1.

maximum EC absorption, near $s=0.5$, the extremity of the resonant ellipses for EC waves, where most of EC power is deposed, occurs for $u_{\|} \simeq 2$, while the LH-produced tail appears for $u_{\|} \geq 3$.

Fig. 10 shows the relative efficiency for two different values of $\alpha$, considering the case of magnetic transport. It is seen that the presence of the barrier (in the case of the figure, with $\alpha=0.125$ ) produces a relative efficiency slightly above the relative efficiency obtained in the case without barrier $(\alpha=$ 1.0). As we have seen in previous figures, the current drive efficiency is increased as effect of transport, in the proposed conditions of good confinement. The presence of the barrier with finite depth, on the other hand, retards the radial diffusion 

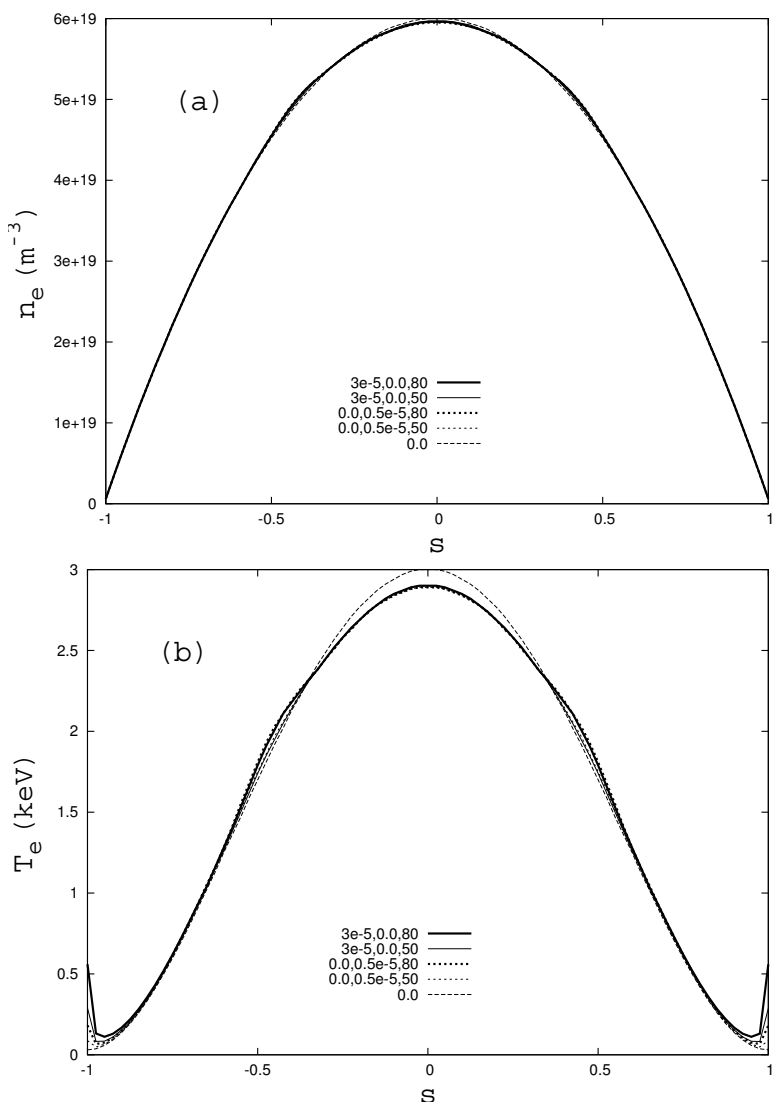

FIG. 11: (a) Electron density as function of position in the plasma slab, at $\tau=50$ and $\tau=80$. (b) Electron temperature as function of position in the plasma slab, at $\tau=50$ and $\tau=80$. The curves show $\widetilde{b}_{0}=3.0 \times 10^{-5}$ and $\widetilde{e}_{0}=0.0$ at $\tau=50$ (thin line), $\widetilde{b}_{0}=3.0 \times 10^{-5}$ and $\widetilde{e}_{0}=0.0$ at $\tau=80$ (thick line), $\widetilde{e}_{0}=0.50 \times 10^{-5}$ and $\widetilde{b}_{0}=0.0$ at $\tau=50$ (thin dotted line), $\widetilde{e}_{0}=0.50 \times 10^{-5}$ and $\widetilde{b}_{0}=0.0$ at $\tau=80$ (thick dotted line). The initial profile is also shown in both panels (thin dashed lines). Other parameters are the same as in Fig. 1.

and allows longer time of interaction between the particle and the waves, therefore increasing the efficiency.

Fig. 11(a) shows the electron density as function of position in the plasma slab, at $\tau=50$ and $\tau=80$, for the cases $\widetilde{b}_{0}=3.0 \times 10^{-5}$ and $\widetilde{e}_{0}=0.0$, and $\widetilde{e}_{0}=0.50 \times 10^{-5}$ and $\widetilde{b}_{0}=0.0$. Fig. 11(b) shows the electron temperature as function of position in the plasma slab, at $\tau=50$ and $\tau=80$, for the case $\widetilde{b}_{0}=3.0 \times 10^{-5}$ and $\widetilde{e}_{0}=0.0$, and $\widetilde{e}_{0}=0.50 \times 10^{-5}$ and $\widetilde{b}_{0}=0.0$. The initial profile is also shown in both panels. Other parameters are the same as in Fig. 1. In the scale of the figure it is not possible to distinguish in Fig. 11(a) any significant modification of the density profile, which is at $\tau=80$ nearly the same as at $\tau=0$, both for magnetic and for electrostatic transport. On the other hand, in Fig. 11(b) it is possible to notice a small decrease of the electron temperature at the plasma center, along with a small increase of the temperature at $s \simeq 0.5$, where both LH and EC wave energy is absorbed, and a more significant increase near the plasma edge, in the cases with transport.
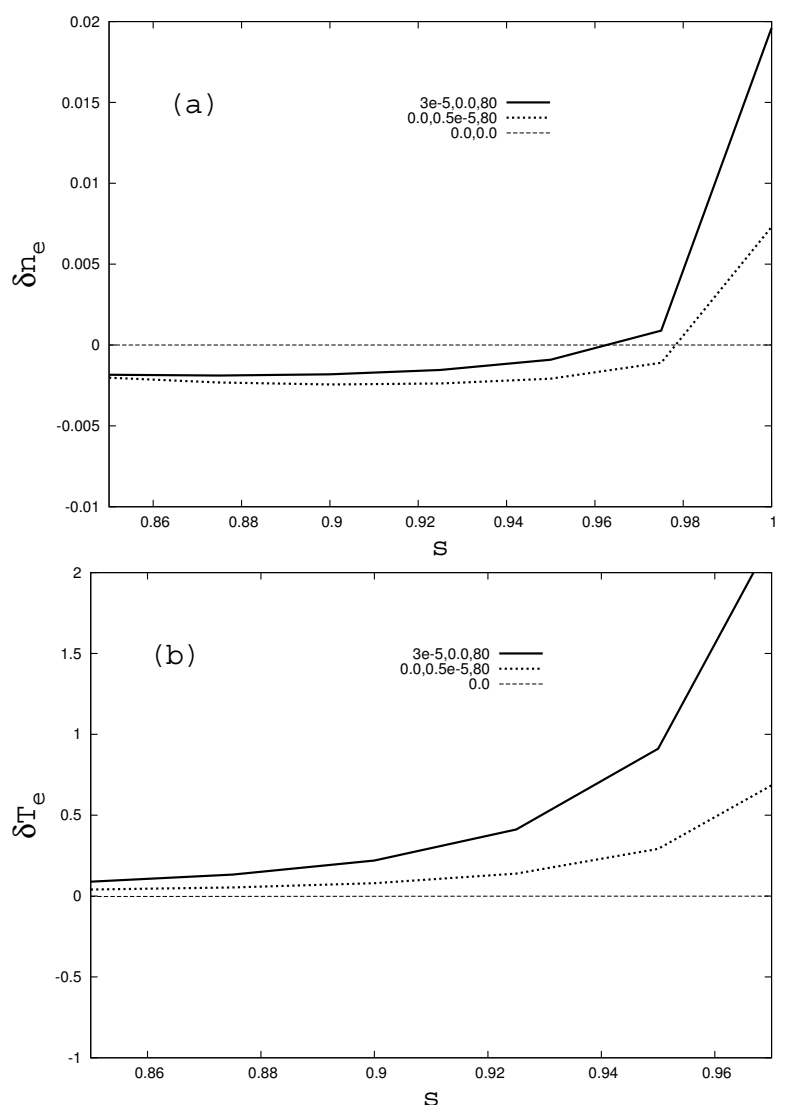

FIG. 12: (a) Amplified vision of the relative variation of the electron density, $\delta n_{e}=\left(n_{e}(\tau)-n_{e}(\tau=0)\right) / n_{e}(\tau=0)$, for positions near the plasma edge, at $\tau=80$. (b) Amplified vision of the relative variation of the electron temperature, $\delta t_{e}=\left(t_{e}(\tau)-t_{e}(\tau=0)\right) / t_{e}(\tau=0)$, for positions near the plasma edge, at $\tau=80$. In both panels the curves show the cases $\widetilde{b}_{0}=3.0 \times 10^{-5}$ and $\widetilde{e}_{0}=0.0$ (thick line), $\widetilde{e}_{0}=0.50 \times$ $10^{-5}$ and $\widetilde{b}_{0}=0.0$ (thick dotted line). Other parameters are the same as in Fig. 1.

An amplified vision of the region near the edge is seen in Fig. 12. Fig. 12a shows the relative variation of the electron density, $\delta n_{e}=\left(n_{e}(\tau)-n_{e}(\tau=0)\right) / n_{e}(\tau=0)$, at $\tau=80$, considering the case of magnetic transport, with $\widetilde{b}_{0}=3.0 \times 10^{-5}$ and $\widetilde{e}_{0}=0.0$, the case of electrostatic transport, with $\widetilde{b}_{0}=0.0$ and $\widetilde{e}_{0}=0.50 \times 10^{-5}$. Fig. $12 \mathrm{~b}$ shows the corresponding figures for the relative variation of the electron temperature, $\delta T_{e}=\left(T_{e}(\tau)-T_{e}(\tau=0)\right) / T_{e}(\tau=0)$, at $\tau=80$. It is seen that the relative variation of density near the edge is nearly $2 \%$ in the case of magnetic transport, and considerably smaller than that in the case of electrostatic transport, while the relative variation of temperature may be nearly $200 \%$ in the case of magnetic transport, and less than $50 \%$ in the case of electrostatic transport. These results confirm the expectation that the transport due to magnetic fluctuations effectively diffuses high velocity particles more efficiently than the transport due to electrostatic fluctuations. Nevertheless, the results obtained show that both types of transport mechanisms produce very similar effects on the combined current drive efficiency. 


\section{CONCLUSIONS}

In this work we have investigated how the occurrence of radial transport and the presence of an Internal Transport Barrier affect the efficiency of current drive by $\mathrm{LH}$ and EC waves. We have considered two possible mechanisms for radial transport, either due to magnetic fluctuations or due to electrostatic fluctuations, and studied the influence of the barrier depth and position and of the level of magnetic or electrostatic perturbation, considering parameters of a medium size tokamak. In our model of the plasma we have used boundary conditions appropriated for regimes where the loss of particles at the plasma edge is very small. Therefore the model is not suitable to describe situations with poor particle confinement. The description of the LH waves assumes a multipass regime, not valid for high-temperature plasmas where single-pass absorption may occur.

Our results have shown some growth of the electron temperature near the plasma edge, more significant in the case of magnetic transport than in the case of transport due to electrostatic fluctuations. Nevertheless, the results show only marginal difference between the effects of the two types of transport mechanism, on the current drive efficiency. The efficiency of the LH+EC current drive has been shown to grow with the level of fluctuations, both in the case of magnetic and electrostatic turbulence, with approximately the same rate of increase. This result can be understood as a consequence of the diffusion of fast particles toward more external regions of the tokamak, where they find smaller density, and consequently longer collisional slowing-down time. The efficiency has been shown to depend weakly on the depth and on the position of the ITB. The minimum of the current drive efficiency has been shown to occur when the barrier is placed close to the position of LH power absorption, but somewhat displaced toward the external edge of the tokamak.

\section{Acknowledgments}

This work has been partially supported by Universidade Federal de Mato Grosso do Sul (UFMS), Conselho Nacional de Desenvolvimento Científico e Tecnológico (CNPq), Fundação de Apoio ao Desenvolvimento do Ensino, Ciência e Tecnologia do Estado de Mato Grosso do Sul (FUNDECT) and Fundação para o Amparo da Pesquisa no Estado do Rio Grande do Sul (FAPERGS). We thank the group of Complex Fluids at UFRGS for granting the use of their cluster of computers for a significant part of the numerical calculations.
[1] C. D. Challis et al., Plasma Phys. Contr. Fusion 43, 861 (2001).

[2] S. J. Wukitch et al., Physics of Plasmas 9, 2149 (2002).

[3] Y. Sakamoto et al., Plasma Phys. Contr. Fusion 44, 876 (2004).

[4] E. J. Doyle et al., Plasma Physics Controlled Fusion 43, A95 (2001).

[5] K. Ushigusa et al., Nuclear Fusion 32, 1977 (1992).

[6] R. W. Harvey, O. Sauter, R. Prater, and P. Nikkola, Phys. Rev. Lett. 88, 205001, 4 pages (2002).

[7] P. R. da S. Rosa and L. F. Ziebell, Plasma Phys. Contr. Fusion 44, 2065 (2002).

[8] C. I. d. Oliveira, L. F. Ziebell, and P. R. da S. Rosa, Plasma Physics Controlled Fusion 47, 249 (2005).

[9] I. Fidone, G. Giruzzi, G. Granata, and R. L. Meyer, Phys. Fluids 27, 2468 (1984).

[10] P. R. da S. Rosa and L. F. Ziebell, Plasma Phys. Contr. Fusion 41, 525 (1999).

[11] R. Dumont, G. Giruzzi, and E. Barbato, Phys. Plasmas 7, 4972 (2000).

[12] E. Barbato, Plasma Phys. Contr. Fusion 40, A63 (1998).

[13] I. Fidone, G. Granata, and R. L. Meyer, Phys. Fluids 25, 2249 (1982).

[14] P. R. da S. Rosa and L. F. Ziebell, Plasma Phys. Contr. Fusion 35, 511 (1993).

[15] P. R. da S. Rosa and L. F. Ziebell, Plasma Phys. Contr. Fusion 38, 375 (1996).
[16] G. Giruzzi, I. Fidone, and X. Garbet, Nuclear Fusion 32, 1011 (1992), report EUR-CEA-FC-1440, DRFC, CEN, Cadarache, France, Nov. 1991.

[17] G. Giruzzi, Plasma Phys. Contr. Fusion 35, A123 (1993).

[18] G. Giruzzi et al., Plasma Phys. Contr. Fusion 38, 1593 (1996).

[19] A. J. Wootton, H. Y. W. Tsui, and S. Prager, Plasma Phys. Contr. Fusion 34, 2023 (1992).

[20] G. G. Castle and A. J. Wootton, IEEE Transactions on Plasma Science 22, 369 (1994).

[21] X. Litaudon et al., Plasma Phys. Contr. Fusion 43, 677 (2001).

[22] C. D. Challis et al., Plasma Phys. Contr. Fusion 44, 1031 (2002).

[23] Y. F. Baranov et al., Plasma Phys. Contr. Fusion 46, 1181 (2004).

[24] P. C. Liewer, Nuclear Fusion 25, 543 (1985).

[25] Y. J. Kim et al., Physics of Fluids B 3, 674 (1991).

[26] T. C. Luce et al., Physical Review Letters 83, 4550 (1999).

[27] M. Shoucri et al., Computer Phys. Communications 55, 253 (1989).

[28] R. J. Dumont and G. Giruzzi, Physics of Plasmas 11, 3449 (2004).

[29] G. Giruzzi et al., Physical Review Letters 93, 255002, 4p. (2004). 